\title{
Decay of Correlations for Certain Quadratic Maps *
}

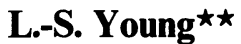

Department of Mathematics, University of California at Los Angeles, Los Angeles, CA 90024, USA

Received June 17, 1991

Abstract. We prove exponential decay of correlations for $(f, \mu)$, where $f$ belongs in a positive measure set of quadratic maps of the interval and $\mu$ is its absolutely continuous invariant measure. These results generalize to other interval maps.

Consider a dynamical system generated by a map $f: M \rightarrow M$ preserving a probability measure $\mu$, and let $\varphi, \psi: M \rightarrow \mathbb{R}$ be observables. Mixing properties of the dynamical system are reflected in the decay of correlations between $\varphi$ and $\psi \circ f^{n}$ as $n \rightarrow \infty$. More precisely, we say that $(f, \mu)$ has exponential decay of correlations for functions belonging in a certain class $X$ if there is a number $\tau<1$ such that for every $\varphi, \psi \in X$, there is a constant $C=C(\varphi, \psi)$ such that

$$
\left|\int \varphi \cdot\left(\psi \circ f^{n}\right) d \mu-\int \varphi d \mu \cdot \int \psi d \mu\right| \leqq C \tau^{n} \quad \forall n \geqq 0 .
$$

The main result of this paper is the following:

Theorem. Consider $f_{a}:[-1,1] \bigcirc$ defined by $f_{a}(x)=1-a x^{2}, a \in[0,2]$. Then there is a positive Lebesgue measure set $\Delta$ in parameter space such that if $f=f_{a}$ for $a \in \Delta$, then

(1) $f$ has an absolutely continuous invariant measure $\mu$ (this is a well known theorem first proved by Yakobson $[\mathrm{J}]$ );

(2) $(f, \mu)$ has exponential decay of correlations for functions of bounded variation;

(3) the central limit theorem holds for $\left\{\varphi \circ f^{n}\right\}_{n=1,2, \ldots}, \varphi \in B V$.

These results generalize to certain open sets of 1-parameter families of unimodal maps.

Exponential decay of correlations has been proved for primarily two types of dynamical systems: piecewise uniformly expanding maps of the interval with their absolutely continuous invariant measures, and Axiom A diffeomorphisms with their Gibbs states. (See e.g. [HK, Ry1, Ru1, Ru2].) These are by no means the only results. (See e.g. [BS], [Z].)

\footnotetext{
$\star$ The results in this paper are announced in the Tagungsbericht of Oberwolfach, June 1990
}

$\star \star$ The author is partially supported by NSF 
The main feature that is new here is that the maps we consider have singularities, and that these singularities return arbitrarily close to themselves. We reduce the problem to the expanding case by constructing a uniformly expanding map $\tilde{f}$ that factors over $f, \tilde{f}$ acting on a set $J$ that is the disjoint union of a countable number of intervals. We then consider a function space on $J$ with a norm that is a weighted combination of the $L^{\infty}, L^{1}$ and total variation norms, and prove the existence of a gap in the spectrum of the Perron-Frobenius operator associated with $\tilde{f}$.

The maps for which our results hold are those studied by Benedicks and Carleson in [BC2]. They have a very simple description, and are non-uniformly expanding in a controlled way. We will recall in detail all of the relevant material from [BC2] - except the proof of the theorem which says that these maps form a positive measure set in parameter space.

This paper is organized as follows. Precise statements of our results are given in Sect. 1. Section 2 contains some background material for 1-dimensional maps. In Sect. 3 we prove the existence of absolutely continuous invariant measures and their mixing properties. Sections 4 and 5 contain the proof of the decay of correlation result.

The author thanks M. Rychlik for helpful conversations.

\section{Statements of Results}

We first state our results for the quadratic family $x \mapsto 1-a x^{2}$ before proceeding to discuss generalizations to other 1-parameter families of interval maps.

Consider $f_{a}:[-1,1] \bigcirc$ defined by $f_{a}(x)=1-a x^{2}, a \in[0,2]$. Let $\alpha>0$ be a very small number that remains fixed throughout, say $\alpha=\frac{1}{10^{6}}$. For $\varepsilon>0$, let

$$
\Delta_{\varepsilon}=\left\{a \in[2-\varepsilon, 2]:\left|f_{a}^{n} 0\right| \geqq e^{-\alpha n} \quad \text { and } \quad\left|\left(f_{a}^{n}\right)^{\prime}\left(f_{a} 0\right)\right| \geqq(1.9)^{n} \forall n \geqq 0\right\} .
$$

Benedicks and Carleson proved that $\operatorname{Leb}\left(\Delta_{\varepsilon}\right)>0 \forall \varepsilon>0$ [BC2]. These are the parameters to which our results apply.

Theorem 1 (Existence of Invariant Measures). $\exists \varepsilon_{1}>0$ such that $\forall a \in \Delta_{\varepsilon_{1}}, f=f_{a}$ has an invariant probability measure $\mu$ with a density $\rho$ that can be written as

$$
\rho=\rho_{1}+\rho_{2},
$$

where $\rho_{1}$ has bounded variation and

$$
0 \leqq \rho_{2}(s) \leqq \text { const } \sum_{j=1}^{\infty} \frac{(1.9)^{-j}}{\sqrt{\left|s-f^{j} 0\right|}} .
$$

The existence of absolutely continuous invariant measures under similar conditions has been proved many times. See e.g. [J, BC1, CE plus N, Ry2, BY], etc. The nature of the density can be understood as follows. The dynamics of $f$ consists essentially of an expanding part and a contraction due to the quadratic singularity. The expanding part gives rise to $\rho_{1}$, while the contraction together with its subsequent iterates account for the inverse square-root singularities in $\rho_{2}$. 
Theorem 2 (Properties of the Absolutely Continuous Invariant Measure). $\exists \varepsilon_{2}>0$, possibly $<\varepsilon_{1}$, such that $\forall a \in \Delta_{\varepsilon_{2}}, f=f_{a}$ has the following properties:

(a) $\mu$ in Theorem 1 is the only absolutely continuous invariant measure;

(b) $(f, \mu)$ is exact;

(c) $\operatorname{supp} \mu=\left[f^{2} 0, f 0\right]$ and $\inf _{x \in\left[f^{2} 0, f 0\right]} \rho(x)>0$.

We remark that $(f, \mu)$ being exact in this setting is equivalent to $\left(f^{n}, \mu\right)$ being ergodic $\forall n>0$. It is also equivalent to the natural extension of $(f, \mu)$ being isomorphic to a Bernoulli shift. (See $[\mathrm{L}]$.)

Theorem 3 (Decay of Correlations). Let $f$ be as in Theorem 2. Then $\exists \tau \in(0,1)$ such that $\forall \varphi, \psi:[-1,1] \rightarrow \mathbb{R}$ with bounded variation, there is a constant $C=C(\varphi, \psi)$ such that

$$
\left|\int \varphi \cdot\left(\psi \circ f^{n}\right) d \mu-\int \varphi d \mu \cdot \int \psi d \mu\right| \leqq C \tau^{n} \forall n \geqq 0 .
$$

Exponential decay of correlations has been proved for various classes of interval maps. The piecewise uniformly expanding case is treated by Hofbauer and Keller [HK] and extended to allow for infinitely many branches by Rychlik [Ry2]. Ziemian [Z] proved the same result for a class of maps satisfying what is sometimes called "the Misiurewicz condition" [M]. Our aim is to relax this condition sufficiently so that our results hold for a positive measure set of parameters in the quadratic and other families.

Our proof of Theorem 3 consists of constructing an extension of $f:([-1,1], \mu) \bigcup$ and analyzing the spectral properties of the Perron-Frobenius operator associated with this extended map. As noted in [K], this knowledge of the spectrum gives us immediately the following theorem.

Theorem 4 (Central Limit Theorem). Let fbe as in Theorem 2, and let $\varphi:[-1,1] \rightarrow$ $\mathbb{R}$ be a function with bounded variation and $\int \varphi d \mu=0$. Then

$$
\sigma:=\frac{1}{\sqrt{n}}\left[\int\left(\sum_{i=0}^{n-1} \varphi \circ f^{i}\right)^{2} d \mu\right]^{1 / 2}
$$

is well defined, and if $\sigma>0$, then $\forall x \in \mathbb{R}$

as $n \rightarrow \infty$.

$$
\mu\left\{\frac{1}{\sqrt{n}} \sum_{i=0}^{n-1} \varphi \circ f^{i}<x\right\} \rightarrow \frac{1}{\sqrt{2 \pi} \sigma} \int_{-\infty}^{x} e^{-u^{2} / 2 \sigma^{2}} d u
$$

Theorem 4 is proved in a similar setting in an unpublished work of Collet [C] using the approach of [BS]. [C] also contains a slightly weaker version of Theorem 3.

We now mention some generalization of our results. Let $I$ be an interval, and let $f_{*}: I \bigcirc$ be a unimodal map satisfying the Misiurewicz condition. That is, $f_{*}$ has negative Schwarzian, it has no sinks, and if $c$ is the critical point, then $\inf \left|f^{n} c-c\right|>0$. Let $\operatorname{End}^{3}(I)$ denote the space of $C^{3}$ endomorphisms of $I$. It is $n>0$

known that there exist constants $\alpha_{*}>0$ and $\lambda_{*}>1$ (depending only on $f_{*}$ ) such that for "most" smooth 1-parameter families $\eta:(-1,1) \rightarrow \operatorname{End}^{3}(I)$ with $\eta(0)=f_{*}$, if $\Delta_{\varepsilon}(\eta)=\left\{a \in(-\varepsilon, \varepsilon) \mid\right.$ if $f=\eta(a)$, then $\left|f^{n} c-c\right| \geqq e^{-\alpha_{*} n} \quad$ and $\left.\quad\left|\left(f^{n}\right)^{\prime}(f c)\right| \geqq \lambda_{*}^{n} \forall n \geqq 1\right\}$, 
then $\operatorname{Leb}\left(\Delta_{\varepsilon}(\eta)\right)>0 \forall \varepsilon>0$. (See [TTY] for details.) Our results carry over directly to $\eta$. That is, Theorem 1 above holds (with different constants), and if $f_{*}$ satisfies (P3) in Sect. 2, then Theorems 2,3 and 4 are valid also.

In the rest of this paper we will consider only the quadratic family $x \mapsto 1-a x^{2}$. This avoids carrying around constants that depend only on $f_{*}$.

\section{Some Properties of 1-Dimensional Maps}

In this section we try to isolate those properties of $f=f_{a}$ that are relevant to our discussion. These are essentially the only properties that will be used.

The specific formula of $f$ is immaterial to us, but we will use the fact that it is a $C^{3}$ unimodal map with negative Schwarzian and nondegenerate critical point. The fact that $f$ is symmetric about 0 simplifies our notation a little, but this is a totally unnecessary assumption. What is important is that $f$ has certain expanding properties, which we summarize below as properties (P1) and (P2).

Let $(-\delta, \delta)$ be a small neighborhood of the critical point. We distinguish between the dynamics of $f$ outside of $(-\delta, \delta)$ and that of orbits beginning in $(-\delta, \delta)$. (P1) concerns the former:

(P1) $\exists M \in \mathbb{Z}^{+}$and $\lambda>1$ such that

(i) if $x, f x, \ldots, f^{M-1} x \notin(-\delta, \delta)$, then $\left|\left(f^{M}\right)^{\prime} x\right| \geqq \lambda^{M}$;

(ii) for any $k \in \mathbb{Z}^{+}$, if $x, \ldots, f^{k-1} x \notin(-\delta, \delta)$ and $f^{k} x \in(-\delta, \delta)$, then $\left|\left(f^{k}\right)^{\prime} x\right| \geqq \lambda^{k}$.

(P1) holds for $f_{a}$ for all $a$ sufficiently near 2 . First notice that it is satisfied by $f_{2}$. One way of seeing this is to conjugate $f_{2}$ to its piecewise linear model. (For a more general approach see $[\mathrm{M}]$ and the appendix of $[\mathrm{CE}]$.) The rest follows because (P1) is an open condition.

For $x \in(-\delta, \delta), f^{\prime} x$ can, of course, be arbitrarily small. (P2) guarantees a period during which $\left(f^{j}\right)^{\prime} x$ recovers. Suppose $\delta=e^{-k_{0}}$. Let $I_{k}=\left(e^{-(k+1)}, e^{-k}\right)$ for $k \geqq k_{0}$, and $-I_{-k}$ for $k \leqq-k_{0}$.

(P2) There is a function $p:(-\delta, \delta)-\{0\} \rightarrow \mathbb{Z}^{+}$, constant on $I_{k}$ and increasing with $|k|$, such that

(i) $\frac{1}{2} k \leqq p(x) \leqq 4 k$ for $x \in I_{ \pm k}$;

(ii) $\left|\left(f^{j}\right)^{\prime}(f x)\right| \approx$ const $\left|\left(f^{j}\right)^{\prime}(f 0)\right| \geqq \operatorname{const}(1 \cdot 9)^{j} \forall j<p(x)$;

(iii) $\left|\left(f^{p}\right)^{\prime} x\right| \geqq \lambda^{p}$ for some $\lambda>1$ (independent of $x$ ).

(P2) holds for all $f_{a}$ with $a \in \Delta_{\varepsilon}, \varepsilon$ sufficiently small. This property is due to Benedicks and Carleson, and the assertion in the last sentence is proved in [BC1] and [BC2]. Since the main construction in our paper is based on this property, let us recall the ideas in their proof:

For $x \neq 0 \in(-\delta, \delta)$, let $p_{0}(x)=\max \left\{p \in \mathbb{Z}:\left|f^{j} x-f^{j} 0\right|<e^{-10 \alpha j} \forall j<p\right\}$. First we claim that $\exists C_{0}$ (independent of $\delta$ ) such that $\forall y \in[f x, f 0]$ and $j<p(x)$,

$$
\frac{1}{C_{0}} \leqq\left|\frac{\left(f^{j}\right)^{\prime} y}{\left(f^{j}\right)^{\prime}(f 0)}\right| \leqq C_{0} .
$$

This is true because the quotient in question is $\sim \exp \sum_{k=1}^{j} \theta(k)$, where

$$
|\theta(k)|=\left|\frac{f^{\prime}\left(f^{k-1} y\right)-f^{\prime}\left(f^{k} 0\right)}{f^{\prime}\left(f^{k} 0\right)}\right| \leqq \frac{e^{-10 \alpha k}}{e^{-\alpha k}} .
$$


Using this distortion estimate, we see that

$$
a x^{2} \cdot \frac{1}{C_{0}}(1.9)^{p_{0}-1} \leqq\left|f^{p_{0}}[0, x]\right| \leqq 1,
$$

giving $p_{0}(x) \leqq 4 k$ for $x \in I_{ \pm k}$ provided $k$ is sufficiently large. The lower bound for $p_{0}(x)$ is obtained from

$$
a x^{2} \cdot 4^{p_{0}-1} \geqq e^{-10 \alpha p_{0}} .
$$

Finally, at time $p_{0}$, we must have

$$
a x^{2} \cdot\left|\left(f^{p_{0}-1}\right)^{\prime} y\right| \geqq e^{-10 \alpha p_{0}}
$$

for some $y \in[f x, f 0]$. This gives

$$
\left|\left(f^{p_{0}}\right)^{\prime} x\right| \geqq 2 a|x| \cdot \frac{1}{C_{0}} \frac{e^{-10 \alpha p_{0}}}{a x^{2}} \geqq \frac{2}{C_{0}} e^{\left(p_{0} / 4\right)-10 \alpha p_{0}},
$$

which is $\geqq \lambda^{p_{0}}$ for some $\lambda>1$ provided $p_{0}$ is reasonably large. For $x \in I_{k}$, let

$$
p(x)=\inf _{y \in I_{k}} p_{0}(y)
$$

The number $p(x)$ is called the "bound period" of $x$. In Sect. 4 we will use the notion of "total bound period," which is defined as follows: For $x \in(-\delta, \delta), \hat{p}(x)$ is the smallest $j>0$ such that $\forall i$ with $0 \leqq i<j$, if $f^{i} x \in(-\delta, \delta)$, then $p\left(f^{i} x\right)<j$. It is easy to see that $\hat{p}(x) \leqq 6 k$ for $x \in I_{ \pm k}$, because if $f^{i} x \in(-\delta, \delta)$ for some $i<p(x)$, then

$$
\begin{aligned}
\left|f^{i} x\right| & \geqq\left|f^{i} 0\right|-\left|f^{i} 0-f^{i} x\right| \\
& \geqq\left|f^{i} 0-f^{i} x\right|^{1 / 9} \\
& \geqq\left[a x^{2} \cdot \frac{1}{C_{0}}(1.9)^{i-1}\right]^{1 / 9} \geqq x^{1 / 4},
\end{aligned}
$$

so $p\left(f^{i} x\right) \leqq k$.

(P1) and (P2) together are sufficient for proving Theorem 1. For Theorems 2-4 we need an additional topological mixing condition:

(P3) For every interval $I \subset[-1,1], \exists n=n(I)$ such that $f^{n} I \supset\left[f^{2} 0, f 0\right]$.

Lemma 2.1. (P3) holds for all $f_{a}$ with $a \in \Delta_{\varepsilon}, \varepsilon$ sufficiently small.

Proof. Let $f=f_{a}, a \in \Delta_{\varepsilon}$. First we prove that $\forall I \subset[-1,1], \exists n_{0}=n_{0}(I)$ such that $f^{n_{0}} I \supset I_{k_{0}}$ or $I_{-k_{0}}$. In light of (P1), some $f^{j} I$ must intersect $(-\delta, \delta)$. If $f^{j} I$ is completely contained in two adjacent $I_{k}$ 's, keep iterating, and note using (P2) that $\left|f^{p}\left(f^{j} I\right)\right| \gg\left|f^{j} I\right|, p=p(x)$ for $x \in f^{j} I$. After finitely many returns to $(-\delta, \delta)$, there must exist $j_{1}$ and $k_{1} \in \mathbb{Z}^{+}$such that $f^{j_{1}} I \supset I_{k_{1}}$ or $I_{-k_{1}}$. Consider $f^{j} I_{k_{1}}, j=1,2, \ldots$, and let $j_{2}$ be the first time (after the bound period of $x \in I_{k_{1}}$ ) such that $f^{j} I_{k_{1}} \supset$ some $I_{k}$. Since $\left|f^{j_{2}} I_{k_{1}}\right| \gg\left|I_{k_{1}}\right|, f^{j_{2}} I_{k_{1}}$ must contain some $I_{k_{2}}$ or $I_{-k_{2}}$ with $0<k_{2}<k_{1}$. We then consider $f^{j} I_{k_{2}}$ and repeat the argument until some $f^{j} I_{k_{n}} \supset I_{k_{0}}$ or $I_{-k_{0}}$.

Next we argue that there is an $n_{1} \in \mathbb{Z}^{+}$such that for all $a$ sufficiently near 2 , if $\hat{x}_{a}$ is the fixed point of $f_{a}$ in $(0,1)$, then $f_{a}^{n_{1}} I_{k_{0}} \ni \hat{x}_{a}$. This is obviously true for $a=2$ and is an open condition. Finally, observe that for $f=$ any $f_{a}$ if $\hat{I}$ is an interval containing $\hat{x}$, then $\exists n_{2}=n_{2}(\hat{I})$ such that $f^{n_{2}} \hat{I} \supset\left[f^{2} 0, f 0\right]$. This completes the proof. 


\section{Existence and Properties of Absolutely Continuous Invariant Measures: Proofs of Theorems 1 and 2}

Our first goal in this section is to prove Theorem 1 assuming (P1) and (P2). The proof is straightforward: Extend $p$ to $p:[-1,1] \rightarrow \mathbb{Z}^{+}$by defining $p(x)=1$ for $x \notin(-\delta, \delta)$. Consider the return map $R:[-1,1] \bigcirc$ given by $R(x)=f^{p(x)}(x)$. Then some power of $R$ is piecewise uniformly expanding. Piecewise uniformly expanding maps have absolutely continuous invariant measures (acim) because their PerronFrobenius operators do not increase total variation by very much. An acim for $f$ is then constructed from that for $R$.

More formally, let $\beta$ be the coarsest partition of $[-1,1]$ into intervals on which $p$ is constant. Define $g:[-1,1] \rightarrow \mathbb{R}$ by

$$
g(x)= \begin{cases}\left|\frac{1}{R^{\prime}(x)}\right| & \text { if } x \in \text { interior }(B), \text { some } B \in \beta \\ 0 & \text { otherwise. }\end{cases}
$$

The Perron-Frobenius operator associated with $R$, written $\mathscr{P}_{R}$ or simply $\mathscr{P}$ when there is no ambiguity, is defined to be

$$
\mathscr{P}(\varphi)(x):=\sum_{y \in R^{-1} x} g(y) \varphi(y) .
$$

Note that if $\beta^{n}:=\beta \vee R^{-1} \beta \vee \cdots \vee R^{-n+1} \beta$, and $g_{n}(x):=g\left(R^{n-1} x\right) \cdots g(x)$, then

$$
\mathscr{P}^{n}(\varphi)(x)=\sum_{y \in R^{-n_{x}}} g_{n}(y) \varphi(y)
$$

Lebesgue measure is denoted by $m$ throughout this paper. $L^{1},\|\cdot\|_{1}$ etc. in this section refer to $L^{1}([-1,1], m)$. If $I$ is an interval, let $V_{I}(\varphi)$ denote the total variation of $\varphi$ on $I$. If $I=[-1,1]$, then we simply write $V(\varphi)$. Say $\varphi \in B V$ if $V(\varphi)<\infty$. It is notationally convenient to assume that each $B \in \beta$ or $\beta^{n}$ is closed, for this allows us to write

$$
V\left(\mathscr{P}^{n} \varphi\right) \leqq \sum_{B \in \beta^{n}} V_{B}\left(\varphi g_{n}\right)=V\left(\varphi g_{n}\right)
$$

Lemma 3.1. (1) $\left\|g_{n}\right\|_{\infty} \rightarrow 0$ as $n \rightarrow \infty$.

(2) $V g_{n}<\infty \forall n$.

Proof. (1) follows immediately from (P1) and (P2). We prove (2) with $n=1$. Let $B=\left[b_{1}, b_{2}\right] \in \beta$. Since $S f<0,\left.g\right|_{\left(b_{1}, b_{2}\right)}$ has exactly one minimum. So

$$
\begin{aligned}
V_{B} g & \leqq 2\left\|g 1_{B}\right\|_{\infty}+\lim _{x \rightarrow b_{1}^{+}} g(x)+\lim _{x \rightarrow b_{2}^{-}} g(x) \\
& \leqq 4\left\|g 1_{B}\right\|_{\infty} .
\end{aligned}
$$

Recall that on $\{p=j\},\left|R^{\prime}\right| \geqq \lambda^{j}$ for some $\lambda>1$. So

$$
V g \leqq V_{[-1,-\delta]} g+V_{[-\delta, 1]} g+8 \sum_{j=1}^{\infty} \lambda^{-j}<\infty .
$$

It is easy to check inductively that $V g<\infty \Rightarrow V g_{n}<\infty \forall n$. 
The idea that the Perron-Frobenius operator shrinks variation for piecewise uniformly expanding maps is due to Lasota and Yorke [LY]. Assuming the additional condition that $V g<\infty$, Rychlik [Ry1] extended their result to the case where $f$ may have an infinite number of uniformly expanding pieces.

Lemma 3.2. (following [Ry1]). Let $\varphi \in B V$. Then

$$
\sup _{n} V\left(\mathscr{P}^{n} \varphi\right)<\infty .
$$

Proof. Choose $N$ such that $\left\|g_{N}\right\|_{\infty}<\frac{1}{10}$. Since the local oscillation of $g_{N}$ at each point is $<\frac{1}{5}$ and $V g_{N}<\infty$, we can choose a finite partition $\beta^{\prime}$ such that

$$
\max _{B \in \beta^{\prime}} V_{B} g_{N}<\frac{1}{4}
$$

Then

$$
\begin{aligned}
V\left(\mathscr{P}^{N} \varphi\right) & \leqq V\left(\varphi g_{N}\right) \\
& =\sum_{B \in \beta^{\prime}} V_{B}\left(\varphi g_{N}\right) \\
& \leqq \sum_{B \in \beta^{\prime}}\left[V_{B} \varphi \cdot\left\|g_{N}\right\|_{\infty}+\left\|\varphi 1_{B}\right\|_{\infty} \cdot V_{B} g_{N}\right] .
\end{aligned}
$$

Using the fact that

$$
\left\|\varphi 1_{B}\right\|_{\infty} \leqq \frac{1}{m(B)}\left|\int_{B} \varphi d m\right|+V_{B} \varphi
$$

we get

$$
V\left(\mathscr{P}^{N} \varphi\right) \leqq \sum_{B \in \beta^{\prime}}\left[V_{B} \varphi \cdot\left(\left\|g_{N}\right\|_{\infty}+V_{B} g_{N}\right)+\frac{V_{B} g_{N}}{m(B)}\left|\int_{B} \varphi d m\right|\right]
$$

which is $<\frac{1}{2} V(\varphi)+C\|\varphi\|_{1}$ with $C=\max _{B \in \beta^{\prime}} \frac{V_{B} g_{N}}{m(B)}$. Since $\|\mathscr{P} \varphi\|_{1} \leqq\|\varphi\|_{1}$, it follows from this that

$$
V\left(\mathscr{P}^{k N} \varphi\right) \leqq\left(\frac{1}{2}\right)^{k} V(\varphi)+\text { const }\|\varphi\|_{1}
$$

for all $k$.

Proof of Theorem 1. Let $\varphi \equiv \frac{1}{2}$. Since $V\left(\frac{1}{n} \sum_{i=0}^{n-1} \mathscr{P}^{n}(\varphi)\right) \leqq$ some $C \forall n$, a subsequence of these functions converges in $L^{1}$ to some $\varphi_{1} \in B V$. Clearly, $\varphi_{1} d m$ is an $R$-invariant probability measure. Let $\mathscr{P}_{f}$ denote the Perron-Frobenius operator associated with $f$, and let

$$
\varphi_{0}:=\sum_{k=0}^{\infty} \mathscr{P}_{f}^{k}\left(\varphi_{1} \cdot 1_{\{p>k\}}\right)
$$

Then $\varphi_{0} d m$ is a finite $f$-invariant measure, finite because $\sum_{k=1}^{\infty}\left\|\varphi_{1}\right\|_{\infty} m\{p>k\}<\infty$ 
(See (P2).) Normalizing $\varphi_{0} d m$, we obtain our desired acim $\mu$. To see that the density of $\mu$ has the properties as claimed, let $\rho_{1}=\varphi_{1} / \int \varphi_{0} d m$. Since

$$
\mathscr{P}_{f}\left(\varphi_{1} \cdot 1_{\{p>1\}}\right)(s) \leqq \frac{\text { const }\left\|\varphi_{1}\right\|_{\infty}}{\sqrt{|s-1|}},
$$

(P2) (ii) tells us that for $k>1$,

$$
\mathscr{P}_{f}^{k}\left(\varphi_{1} \cdot 1_{\{p>k\}}\right)(s) \leqq \frac{\text { const }\left\|\varphi_{1}\right\|_{\infty}(1.9)^{-(k-1)}}{\sqrt{\left|s-f^{k} 0\right|}} .
$$

Next we prove Theorem 2 assuming (P1)-(P3). Note that (P1) and (P2) imply that $f$ has a positive Lyapunov exponent at $\mu$-a.e. $x$. First we recall a theorem of Ledrappier:

Theorem [L]. Let $h:[-1,1] \bigcirc$ be a $C^{1+\varepsilon}$ piecewise monotonic map with nonflat critical points, and let $v$ be an acim with a positive Lyapunov exponent a.e. Then a.e. ergodic component of $v$ is an acim. Moreover, if $\left(h^{n}, v\right)$ is ergodic $\forall n>0$, then the natural extension of $(h, v)$ is isomorphic to a Bernoulli shift.

Our next proposition probably has some independent interest. It is a corollary to the proofs in [L].

Proposition 3.3. Let $h$ : $[-1,1] \bigcirc$ be a $C^{1+\varepsilon}$ piecewise monotonic map with $k$ critical points, all of which are nonflat. Let $v$ be an acim with a positive Lyapunov exponent a.e. Then

(1) $v$ is the sum of at most $k$ ergodic acim's;

(2) each ergodic component of $v$ is supported on a finite number of intervals, on which the density is $>0$ a.e.;

(3) if $h$ is topologically mixing, then $(h, v)$ is measure-theoretically mixing, and hence Bernoulli.

Proof of Proposition 3.3. Let $J_{0}, \ldots, J_{k}$ be the intervals of monotonicity of $h$. It is shown in [L] that if we view the natural extension $(\bar{h}, \bar{v})$ of $(h, v)$ as living on

$$
\begin{gathered}
\Omega=\left\{(x, \underline{a}) \in[-1,1] \times\{0, \ldots, k\}^{\mathbf{N}} \mid \exists x_{0}, x_{1}, \ldots \in[-1,1] \text { with } x_{0}=x,\right. \\
\left.h\left(x_{i}\right)=x_{i-1} \text { and } x_{i} \in J_{a_{i}}\right\},
\end{gathered}
$$

then local unstable manifolds of $\bar{h}$ are canonically identified with subintervals of $[-1,1]$ and acim's on $[-1,1]$ with positive Lyapunov exponents correspond to measures on $\Omega$ with smooth conditional measures on unstable manifolds.

Let $v_{i}$ be an ergodic component of $v$. It follows from the discussion above that at $v_{i}$-a.e. $x$, there is an interval $J_{x}^{i}$ containing $x$ on which the density of $v_{i}$ is $>0$ a.e. Let $J_{1}^{i}, J_{2}^{i}, \ldots$ be the maximal intervals on which the density of $v_{i}$ is $>0$ a.e. By the ergodicity of $v_{i}$, there can only be a finite number of these. Moreover, it is not possible for $h \mid J_{j}^{i}$ to be $1-1$ for all $j$, because $h$ has a positive Lyapunov exponent and the $J_{j}^{i}$,s cannot grow indefinitely in length. So at least one of them must get "folded," i.e. $\exists j=j(i)$ such that $J_{j}^{i}$ contains a critical point in its interior. This puts a bound on the number of ergodic components.

Assertion (3) follows from what we have said and the last statement in the theorem we quoted from [L]. 
Proof of Theorem 2. Since $\rho_{1} \in B V$, there is an interval $I \subset[-1,1]$ such that $\inf \rho(x)>0$. By virtue of $(\mathrm{P} 3)$, we have inf $\rho(x)>0$. The rest follows immediately $x \in I$ from Proposition 3.3 and (P3).

\section{Decay of Correlations: Main Steps in the Proof of Theorem 3}

Let $f$ be as in Theorem 2 .

Step I. Construction of an extension of $f:([-1,1], \mu) \bigcirc$. Recall that there is a function $p:[-1,1]-\{0\} \rightarrow \mathbb{Z}^{+}$with the following properties:

$* p(x) \equiv 1 \forall x \notin(-\delta, \delta)$,

* $\frac{1}{2} k \leqq p(x) \leqq 4 k \forall x \in I_{ \pm k}$,

$* p(x) \uparrow$ as $|x| \downarrow 0$,

$* \exists \lambda>1$ such that $\forall x \neq 0 \in(-\delta, \delta),\left|\left(f^{p}\right)^{\prime} x\right| \geqq \lambda^{p}$.

For reasons to become obvious shortly, we will choose $\lambda$ with $\lambda e^{-1 / 4}<1$.

Our new space $J$ is the disjoint union of a countable number of intervals $J_{1}, J_{2}, \ldots$, where $J_{1}=[-1,1]$ and for $k>1, J_{k}$ has length $\lambda^{(k-1) / 2} m\{p \geqq k\}$. Note immediately that

$$
\sum_{1}^{\infty} m\left(J_{k}\right) \leqq 1+\sum_{k>1} \lambda^{(k-1) / 2} \cdot 2 \min \left\{\delta, e^{-k / 4}\right\}<\infty .
$$

We now define a map $\tilde{f}: J り$. Let

$$
J_{1}^{-}=[-1,-\delta], \quad J_{1}^{0}=(-\delta, \delta), \quad J_{1}^{+}=[\delta, 1] .
$$

On $J_{1}^{-} \cup J_{1}^{+}$, let $\tilde{f}=f$ in the sense that $f\left(J_{1}^{ \pm}\right) \subset J_{1}$. The interval $J_{1}^{0}$ is mapped affinely onto $J_{2}$, with a magnification of $\lambda^{1 / 2}$. Assume now that $\tilde{f}$ has been defined on $J_{1}, \ldots, J_{k-1}$. We again write $J_{k}=J_{k}^{-} \cup J_{k}^{0} \cup J_{k}^{+}$, where $J_{k}^{0}=\tilde{f}^{k-1}\{p>k\}$. The map $\tilde{f}$ then takes $J_{k}^{0}$ affinely onto $J_{k+1}$, magnifying it by $\lambda^{1 / 2}$, and takes $J_{k}^{ \pm}$into $J_{1}$ in such a way that $\forall x \in\{p=k\} \subset J_{1}^{0}, \tilde{f}^{p} x=f^{p} x$. Note that on $J_{k}^{ \pm},\left|\tilde{f}^{\prime}\right| \geqq \lambda^{k / 2}$.

Clearly, there is a projection $\pi: J \rightarrow[-1,1]$ such that $\pi \circ \tilde{f}=f \circ \pi$.

Next observe that $\mu$ lifts to an $\tilde{f}$-invariant probability measure $\tilde{\mu}$ on $J$ with density

$$
\tilde{\rho}:=\sum_{k=1}^{\infty} \mathscr{P}_{\tilde{f}}^{k}\left(\rho_{1} \cdot 1_{\{p>k\}}\right)
$$

where $\rho_{1}$ is as in Theorem 1. We mention a few relevant properties of $\tilde{\rho}$. For a function $\varphi: J \rightarrow \mathbb{R}$ or $\mathbb{C}$, let us use the notation $\varphi_{k}$ to denote $\left.\varphi\right|_{J_{k}}$. Then $\tilde{\rho}_{k} \in B V \forall k$ and $\left\|\tilde{\rho}_{k}\right\|_{\infty} \leqq \lambda^{-(k-1) / 2}\left\|\tilde{\rho}_{1}\right\|_{\infty}$. Since $p>1$ on $(-\delta, \delta)$, the support of $\tilde{\rho}$ is contained in $\left[f^{2} \delta, f \delta\right] \cup \bigcup_{k>1} J_{k}$. Henceforth let us change $J_{1}$ to $\left[f^{2} \delta, f \delta\right]$. Moreover, if $I \subset J_{1}$ is an interval, then $\exists n=n(I)$ such that $\tilde{f}^{n} I$ contains the fixed point of $\tilde{f}$ in $J_{1}^{+}$. (This follows from the corresponding statement for $f$.) So the same argument as in the last section tells us that inf $\tilde{\rho}_{1}(x)>0$.

$$
x \in J_{1}
$$

Lemma 4.1. $(\tilde{f}, \tilde{\mu})$ is exact.

Proof. Let $\tilde{\beta}$ be the partition of $J$ into $\left\{J_{k}^{ \pm, 0}\right\}$. We have $H_{\tilde{\mu}}(\tilde{\beta})<\infty$ because 
const $e^{-2 k} \leqq \tilde{\mu}\left(J_{k}^{0}\right) \leqq$ const $e^{-k / 4} \forall k$, and $\tilde{\mu}\left(J_{k}^{ \pm}\right)$is either 0 or has similar bounds. Since $\tilde{f}$ is essentially expanding, $\tilde{\beta}$ is clearly a generator. So if $\tilde{\Gamma}:(\overline{\tilde{f}}, \tilde{\tilde{\mu}}) \rightarrow(\tilde{f}, \tilde{\mu})$ is the natural extension of $(\tilde{f}, \tilde{\mu})$, and $\mathscr{B}$ is the Borel $\sigma$-algebra on $J$, then $\tilde{\Gamma}^{-1}\left(\underset{n \geqq 0}{\wedge} \tilde{f}^{-n} \mathscr{B}\right)$ is contained in the Pinsker $\sigma$-algebra of $(\overline{\tilde{f}}, \overline{\tilde{\mu}})([\mathrm{Ro}]$ 12.3). It therefore suffices to prove the triviality of the Pinsker $\sigma$-algebra of $(\overline{\tilde{f}}, \overline{\tilde{\mu}})$.

We will show that $(\overline{\tilde{f}}, \overline{\tilde{\mu}})$ is measure-theoretically isomorphic to $(\bar{f}, \bar{\mu})$, the natural extension of $(f, \mu)$. (See Theorem 2.) Let $\underline{\tilde{x}}=\left(\tilde{x}_{0}, \tilde{x}_{1}, \ldots\right)$ be a history of $(\tilde{f}, \tilde{\mu})$ and define $\Phi(\underline{\bar{x}})=\underline{x}=\left(x_{0}, x_{1}, \ldots\right)$ by letting $x_{i}=\pi \tilde{x}_{i}$. Clearly $\Phi_{*}(\overline{\tilde{\mu}})=\bar{\mu}$, since $\Phi_{*}(\overline{\tilde{\mu}})$ is $\bar{f}$-invariant and projects onto $\mu$. So all we have to do is to verify that $\Phi$ is $1-1$ a.e..

Let $\underline{x}=\left(x_{0}, x_{1}, \ldots\right)$ be a typical element of $(\bar{f}, \bar{\mu})$. We say that $x_{i}$ is a "marker" for $\underline{x}$ if $x_{i} \in(-\delta, \delta)$ and $\exists j>i$ such that $\hat{p}\left(x_{j}\right)>j-i, \hat{p}$ being the total bound period defined in Sect. 2. If $x_{i}$ is a marker, and $\Phi(\tilde{\tilde{x}})=\underline{x}$, then the only possibility for $\tilde{x}_{i}$ is that $\tilde{x}_{i}=x_{i} \in J_{1}$. Hence $\Phi^{-1}\{\underline{x}\}$ contains at most one point if $\underline{x}$ has infinitely many markers.

Assume for simplicity that $x_{0} \in(-\delta, \delta)$. From Theorem 1 we know that $\Sigma_{j} \mu\left(-e^{-j / 6}, e^{-j / 6}\right)<\infty$, so by the Borel-Cantelli Lemma, $\exists k(\underline{x}) \in \mathbb{Z}^{+}$such that $x_{j} \notin\left(-e^{-j / 6}, e^{-j / 6}\right) \forall j>k(\underline{x})$. This guarantees that for $j>k(\underline{x}), \hat{p}\left(x_{j}\right)<j$. Suppose that $x_{0}$ is not a marker. Let $j_{1}>0$ be such that $x_{j_{1}} \in(-\delta, \delta)$ and $p\left(x_{j_{1}}\right)>j_{1}$. Then either $x_{j_{1}}$ is a marker, or $\exists j_{2}>j_{1}$ such that $x_{j_{2}} \in(-\delta, \delta)$ and $p\left(x_{j_{2}}\right)>j_{2}-j_{1}$. If this process continued, there would be a $j_{n}>k(\underline{x})$ such that $\hat{p}\left(x_{j_{n}}\right)>j_{n}$, contradicting our choice of $k(\underline{x})$.

Step II. The Perron-Frobenius operator. For the rest of this paper let $m$ denote Lebesgue measure on $J$, and let $\|\cdot\|_{1},\|\cdot\|_{\infty}$ denote the $L^{1}$ and $L^{\infty}$ norms with respect to $m$ on $J$. For $\varphi: J \rightarrow \mathbb{C}$, we define

$$
V(\varphi)=\sum_{k} V\left(\varphi_{k}\right)
$$

where $V\left(\varphi_{k}\right)$ is the total variation of $\varphi$ on $J_{k}$. Consider the function space

$$
X=\left\{\varphi: J \rightarrow \mathbb{C} \mid V(\varphi)<\infty,\|\varphi\|_{1}<\infty,\|\varphi\|_{\infty}<\infty\right\}
$$

with norm

$$
\|\varphi\|:=V(\varphi)+\|\varphi\|_{1}+\varepsilon\|\varphi\|_{\infty},
$$

where $\varepsilon>0$ is a small number to be determined later. $(X,\|\cdot\|)$ is a Banach space.

As usual, the Perron-Frobenius operator $\tilde{\mathscr{P}}$ associated with $\tilde{f}$ is defined to be

$$
\tilde{\mathscr{P}}(\varphi)=\sum_{y \in \tilde{f}^{-1} x} \varphi(y) \tilde{g}(y),
$$

where

$$
\tilde{g}(y)=\frac{1}{\left|\tilde{f}^{\prime} y\right|}
$$

except possibly at the end points of $\widetilde{\beta}=\left\{J_{k}^{ \pm, 0}\right\}$. For purposes of estimating variation it is convenient to adopt the following convention. For $B \in \widetilde{\beta}^{n}$ and $a \in \partial B$, we consider $a$ as belonging in $B$ if for some $j, 0 \leqq j<n, \tilde{f}^{j} B \subset$ some $J_{k}^{ \pm}$and $\tilde{f}^{j} a \in \partial J_{k}^{ \pm}$; otherwise we say $a \notin B$. The advantage of this convention is that if we let $\tilde{g}=0$ on $\partial J_{k}^{ \pm}$and 
$\tilde{g}_{n}(x)=\tilde{g}\left(\tilde{f}^{n-1} x\right) \cdots \tilde{g}(x)$, then we can estimate $V\left(\tilde{\mathscr{P}}^{n} \varphi\right)$ by

$$
V\left(\tilde{\mathscr{P}}^{n} \varphi\right) \leqq \sum_{B \in \tilde{\beta}^{n}} V_{B}\left(\varphi \tilde{g}_{n}\right)
$$

(which is clear), and at the same time have

$$
\sum_{B \in \tilde{\beta}^{n}} V_{B}\left(\tilde{g}_{n}\right)<\infty
$$

(See Sublemma 5.1 and cf. [Ry1].)

The next lemma contains the main estimates in this paper. Its proof is postponed to Sect. 5.

Lemma 4.2. $\tilde{\mathscr{P}}: X \rightarrow X$ is a bounded linear operator whose spectrum

$$
\sigma(\tilde{\mathscr{P}})=\sigma_{0} \cup\left\{\xi_{1}, \ldots, \xi_{n}\right\}
$$

where $\sigma_{0} \subset\left\{|z| \leqq \tau_{0}\right\}$ for some $\tau_{0}<1$ and $\xi_{i} \in S^{1} \forall i$. Moreover, each $\xi_{i}$ is a simple pole, and the corresponding projection has a finite dimensional range.

Step III. Finishing the proof. First we use the exactness of $(\tilde{f}, \tilde{\mu})$ to prove that $\sigma(\tilde{\mathscr{P}}) \cap S^{1}=\{1\}$. Let $\varphi \in X$ be such that $\tilde{\mathscr{P}}(\varphi)=\xi \varphi$ for some $\xi \in S^{1}$. Since $\tilde{\rho}>0$, we can write $\varphi=\theta \tilde{\rho}$ for some $\theta$. Note that $\theta \in L^{2}(\tilde{\mu})$, for

so that

$$
\left\|\theta_{k}\right\|_{\infty} \leqq \frac{\|\varphi\|_{\infty}}{\min \tilde{\rho}_{k}} \leqq\|\varphi\|_{\infty} \cdot \frac{\lambda^{(k-1) / 2}}{\min \tilde{\rho}_{1}},
$$

$$
\int \theta^{2} d \tilde{\mu} \leqq \operatorname{const} \sum_{k} \lambda^{k} e^{-k / 4}
$$

which is $<\infty$ by the upper bound we imposed on $\lambda$.

The rest of this argument is quite standard (see e.g. [HK]). We let $U=U_{\bar{f}}$ be the operator on $L^{2}(\tilde{\mu})$ defined by $U(\psi)=\psi \circ \tilde{f}$. Then $U^{*} \theta=\xi \theta$ (in the sense of $L^{2}$ ) because

$$
\left\langle U^{*} \theta, \psi\right\rangle=\int(\bar{\psi} \circ \tilde{f}) \theta \tilde{\rho} d m=\int \bar{\psi} \cdot \tilde{\mathscr{P}}(\theta \tilde{\rho}) d m=\left\langle\frac{\tilde{\mathscr{P}}(\theta \tilde{\rho})}{\tilde{\rho}}, \psi\right\rangle=\langle\xi \theta, \psi\rangle
$$

for every $\psi \in L^{2}(\tilde{\mu})$. From this and $|\xi|=1$ we deduce that $U \theta=\bar{\xi} \theta$, which means that $\theta=\xi^{n} U^{n} \theta$ is measurable with respect to $\tilde{f}^{-n} \mathscr{B}$. Hence $\theta=$ some constant $c$ a.e. by Lemma 4.1. Thinking of $\widetilde{P}$ as an operator on $L^{1}(m)$, one sees immediately that $\xi=1$.

We have shown that $\varphi=c \tilde{\rho}$ a.e. To see that $\varphi=c \tilde{\rho}$ as elements of $X$, use Lemma 5.1. This together with Lemma 4.2 proves that $\tilde{\mathscr{P}}=\tilde{\mathscr{P}}_{0}+\tilde{\mathscr{P}}_{1}$, where $\tilde{\mathscr{P}}_{0} \tilde{\mathscr{P}}_{1}=$ $\widetilde{\mathscr{P}}_{1} \widetilde{\mathscr{P}}_{0}=0, \sigma\left(\widetilde{\mathscr{P}}_{0}\right) \subset\left\{|z| \leqq \tau_{0}\right\}$, and $\widetilde{\mathscr{P}}_{1}(\varphi)=c_{\varphi} \tilde{\rho}$ for some $c_{\varphi} \in \mathbb{C}$. In fact, $c_{\varphi}=\int \varphi d m$, because $\int \tilde{\mathscr{P}}_{1} \varphi d m=\int \tilde{P}_{1}^{n} \varphi d m \stackrel{\text { large } n}{\approx} \int \tilde{P}^{n} \varphi d m=\int \varphi d m$.

Finally we return to the original dynamical system $f:([-1,1], \mu) \circlearrowleft$. Observe that $\varphi \in B V([-1,1])$ lifts to $\tilde{\varphi}: J \rightarrow \mathbb{R}$ with $\tilde{\varphi} \tilde{\rho} \in X:$

$$
\begin{aligned}
V(\tilde{\varphi} \tilde{\rho}) & \leqq \sum_{k}\left[V\left(\tilde{\varphi}_{k}\right)\left\|\hat{\rho}_{k}\right\|_{\infty}+V\left(\tilde{\rho}_{k}\right)\left\|\tilde{\varphi}_{k}\right\|_{\infty}\right] \\
& \leqq \sum_{k}\left[2 V(\varphi) \cdot \lambda^{-(k-1) / 2}\left\|\tilde{\rho}_{1}\right\|_{\infty}+\lambda^{-(k-1) / 2} V\left(\tilde{\rho}_{1}\right) \cdot\|\varphi\|_{\infty}\right]<\infty
\end{aligned}
$$


We have thus shown that $\forall \varphi, \psi \in B V([-1,1])$,

$$
\begin{aligned}
\left|\int\left(\psi \circ f^{n}\right) \varphi d \mu-\int \varphi d \mu \cdot \int \psi d \mu\right| & =\left|\int\left(\tilde{\psi} \circ \tilde{f}^{n}\right) \tilde{\varphi} d \tilde{\mu}-\int \tilde{\varphi} d \tilde{\mu} \cdot \int \tilde{\psi} d \tilde{\mu}\right| \\
& =\left|\int \tilde{\psi}\left[\tilde{P}^{n}(\tilde{\varphi} \tilde{\rho})-\left(\int \tilde{\varphi} \tilde{\rho} d m\right) \tilde{\rho}\right] d m\right| \\
& \leqq m(J) \cdot\|\psi\|_{\infty} \cdot C(\varphi, \psi) \tau^{n} \quad \text { for some } \tau_{0}<\tau<1 .
\end{aligned}
$$

This completes the proof.

\section{Spectral Properties of the Perron-Frobenius Operator}

The purpose of this section is to prove Lemma 4.2. Recall that our norm consists of a total variation part, an $L^{1}$-part, and an $L^{\infty}$-part. Roughly speaking, the variation part is contracted by $\tilde{\mathscr{P}}$; the $L^{1}$-part does no harm; and the $L^{\infty}$-part is needed to control what happens on the $J_{k}$ 's for $k$ large.

From here on we will be working exclusively with $\tilde{f}: J \sqcup$. So for notational simplicity let us drop all the "s in $\tilde{f}, \tilde{\beta}, \tilde{P}$ etc. The following line of argument is also fairly standard:

Lemma 5.1. (1) $\tilde{\mathscr{P}}$ is a bounded operator on $X$;

(2) $\exists N \in \mathbb{Z}^{+}$and $R \geqq 0$ such that $\forall \varphi \in X$,

$$
\left\|\mathscr{P}^{N} \varphi\right\| \leqq \frac{1}{5}\|\varphi\|+R\|\varphi\|_{1} .
$$

Using Lemma 5.1, and remembering that $\|\mathscr{P} \varphi\|_{1} \leqq\|\varphi\|_{1}$, one deduces immediately that $\sup _{n}\left\|\mathscr{P}^{n}\right\|<\infty$. This gives $\sigma(\mathscr{P}) \subset\{|z| \leqq 1\}$.

Lemma 5.2. For $N$ sufficiently large, there is a finite rank operator $\mathscr{Q}$ with $\left\|\mathscr{P}^{N}-\mathscr{Q}\right\|<1$.

This second lemma tells us that outside of some disk of radius $<1, \sigma(\mathscr{P})$ consists of at most a finite number of points $\eta_{1}, \ldots, \eta_{l}$, and that the projection corresponding to each $\eta_{i}$ has a finite dimensional range ([DS] VIII 8). No $\eta_{i} \in S^{1}$ can be a pole of order $>1$, for that would violate $\sup _{n}\left\|\mathscr{P}^{n}\right\|<\infty$. This completes the proof.

Sublemma 5.1a. $\sup _{B \in \beta^{N}} V_{B}\left(g_{N}\right)<\infty \forall N$.

Proof. Since $\left|f^{\prime} x\right| \geqq \delta \forall x$ and $f$ maps each element of $\beta$ to at most 3 elements of $\beta$, we have

$$
\sum_{\substack{B \in \beta^{N} \\ B \subset J_{1}}} V_{B}\left(g_{N}\right) \leqq 3^{N} \cdot 4 \delta^{-N} .
$$

(See the proof of Lemma 3.1.) Next consider $B \in \beta^{N}$ with $B \subset J_{k}, k>1$. If $f^{j} B \subset$ $\left(\bigcup_{i>1} J_{i}\right) \forall j \leqq N$, then $V_{B} g_{N}=0$. If not, then $\left|\left(f^{N}\right)^{\prime} x\right| \geqq \delta^{N} \lambda^{(k-1) / 2} \forall x \in B$, giving

$$
\sum_{\substack{B \in \beta^{n} \\ B \subset J_{k}}} V_{B}\left(g_{N}\right) \leqq 3^{N} \cdot 4 \delta^{-N} \cdot \lambda^{-(k-1) / 2} .
$$

We now choose and fix an $N \in \mathbb{Z}^{+}$with $\left\|g_{N}\right\|_{\infty} \leqq \frac{1}{100}$ and $\lambda^{-N / 2} \leqq \frac{1}{10}$. 
Sublemma 5.1b. For every $\varepsilon>0, \exists R_{1}=R_{1}(N, \varepsilon)$ such that $\forall \varphi \in X$,

$$
V\left(\mathscr{P}^{N} \varphi\right) \leqq \frac{1}{10} V(\varphi)+R_{1}\|\varphi\|_{1}+\frac{1}{10} \varepsilon\|\varphi\|_{\infty} .
$$

Proof.

$$
V\left(\mathscr{P}^{N} \varphi\right) \leqq \sum_{B \in \beta^{N}} V_{B}\left(\varphi g_{N}\right) \leqq \sum_{B}\left\|g_{N}\right\|_{\infty} V_{B}(\varphi)+\sum_{B}\left\|\varphi 1_{B}\right\|_{\infty} V_{B}\left(g_{N}\right)
$$

The first term is $\leqq\left\|g_{N}\right\|_{\infty} V(\varphi)$, and the second term is estimated as follows. For $K \in \mathbb{Z}^{+}$,

$$
\begin{aligned}
& \sum_{\substack{B \in \beta^{N} \\
B \subset \bigcup_{k \leqq K} J_{k}}} V_{B}\left(g_{N}\right) \cdot\left\|\varphi 1_{B}\right\|_{\infty} \\
& \leqq \sum V_{B}\left(g_{N}\right)\left\{\frac{1}{m B}\left|\int_{B} \varphi d m\right|+V_{B}(\varphi)\right\} \\
& \leqq\left(\max _{B} V_{B}\left(g_{N}\right)\right) \cdot\left(\sum_{B} V_{B}(\varphi)\right)+\left(\max _{B} \frac{V_{B}\left(g_{N}\right)}{m B}\right) \cdot\left(\sum_{B}\left|\int_{B} \varphi\right|\right) \\
& \leqq\left(4\left\|g_{N}\right\|_{\infty}\right) \cdot V(\varphi)+R_{1, K}\|\varphi\|_{1} \text {, }
\end{aligned}
$$

where $R_{1, K}$ is a constant depending on $K$. Choose $K$ such that

$$
\sum_{\substack{B \in \beta^{N} \\ B \subset \bigcup_{k>K^{J_{k}}}}} V_{B}\left(g_{N}\right)<\frac{1}{10} \varepsilon
$$

Sublemma 5.1c. $\forall$ sufficiently small $\varepsilon>0, \exists R_{2}=R_{2}(N, \varepsilon)$ such that

$$
\varepsilon\left\|\mathscr{P}^{N} \varphi\right\|_{\infty} \leqq \frac{1}{10} V(\varphi)+R_{2}\|\varphi\|_{1}+\frac{1}{10} \varepsilon\|\varphi\|_{\infty} .
$$

Let us explain the idea of the lemma, assuming $N=1$. A formal proof will follow. We wanted to say that $\|\mathscr{P} \varphi\|_{\infty}<\|\varphi\|_{\infty}$. Clearly, $\left\|(\mathscr{P} \varphi) \cdot 1 \bigcup_{k>1} J_{k}\right\|_{\infty} \leqq \lambda^{-1 / 2}\|\varphi\|_{\infty}$, but for $(\mathscr{P} \varphi)_{1}$ all we can say is that $\left\|(\mathscr{P} \varphi)_{1}\right\|_{\infty} \leqq C\left\|\varphi_{1}\right\|_{\infty}+2 \sum_{k>1}^{\infty} \lambda^{-(k-1) / 2}\left\|\varphi_{k}\right\|_{\infty}$ for some $C$. Choose $K_{0}$ such that $2 \sum_{k>K_{0}} \lambda^{-(k-1) / 2}$ is small. For $1<k \leqq K_{0}$, we can write $\left\|\varphi_{k}\right\|_{\infty} \leqq V\left(\varphi_{k}\right)+\left|\int \varphi_{k}\right| / m\left(J_{k}\right)$. Together this gives us

$$
\|\mathscr{P} \varphi\|_{\infty} \leqq \text { const } \cdot\left(V(\varphi)+\|\varphi\|_{1}\right)+\left(\lambda^{-1 / 2}+\text { small }\right) \cdot\|\varphi\|_{\infty}
$$

which explains the $\varepsilon$-weight in the $L^{\infty}$-part of the norm $\|\cdot\|$.

Proof of Sublemma 5.1c. Since

$$
\sup _{k>N}\left\|\left(\mathscr{P}^{N} \varphi\right)_{k}\right\|_{\infty} \leqq \lambda^{-N / 2}\|\varphi\|_{\infty} \leqq \frac{1}{10}\|\varphi\|_{\infty}
$$

we only have to consider $\left(\mathscr{P}^{N} \varphi\right)_{k}$ for $k=1, \ldots, N$. Let $N_{0}$ be a large number to be 
specified shortly. Define

$$
\begin{aligned}
& M_{1}(\varphi)=2\left(\sum_{k>N_{0}} \lambda^{-k / 2}\right)\|\varphi\|_{\infty}, \\
& M_{2}(\varphi)=\max \left\{\left\|\varphi_{1}\right\|_{\infty}, \ldots,\left\|\varphi_{N_{0}}\right\|_{\infty}\right\}
\end{aligned}
$$

and

$$
M=\max \left\{M_{1}, M_{2}\right\} .
$$

One verifies inductively that $\forall j, k \leqq N,\left\|\left(\mathscr{P}^{j} \varphi\right)_{k}\right\|_{\infty} \leqq D^{j} M$, where

$$
D=\frac{1}{a \delta}+2 N+2 \sum \lambda^{-k / 2} \text {. }
$$

For instance,

$$
\left\|\left(\mathscr{P}^{j+1} \varphi\right)_{1}\right\|_{\infty} \leqq \frac{1}{a \delta}\left(D^{j} M\right)+2 N\left(D^{j} M\right)+\left(2 \sum \lambda^{-k / 2}\right) M,
$$

the three terms being contributions from $\left(\mathscr{P}^{j} \varphi\right)_{1}, \sum_{2}^{j}\left(\mathscr{P}^{j} \varphi\right)_{i}$ and $\sum_{i>j}\left(\mathscr{P}^{j} \varphi\right)$, respectively.
We have proved that

$$
\left\|\mathscr{P}^{N} \varphi\right\|_{\infty} \leqq \max \left\{\frac{1}{10}\|\varphi\|_{\infty}, D^{N} M(\varphi)\right\} .
$$

If $\frac{1}{10}\|\varphi\|_{\infty}$ is bigger, we are done. So suppose $D^{N} M(\varphi)$ dominates. We choose $N_{0}$ and $\varepsilon$ such that

$$
D^{N}\left(2 \sum_{k>N_{0}} \lambda^{-k / 2}\right) \leqq \frac{1}{10} \text { and } 100 \varepsilon D^{N} \leqq \frac{1}{100}
$$

and consider the following possibilities:

Case 1: $M=M_{1}$. We have $\left\|\mathscr{P}^{N} \varphi\right\|_{\infty} \leqq D^{N} M_{1} \leqq \frac{1}{10}\|\varphi\|_{\infty}$.

Case 2: $M \leqq 100 V(\varphi)$. We have $\varepsilon\left\|\mathscr{P}^{\bar{N}} \varphi\right\|_{\infty} \leqq \frac{1}{100} V(\varphi)$.

Case 3: $M=M_{2}$ and $M_{2}>100 V(\varphi)$. Let $k \leqq N_{0}$ be such that $\left\|\varphi_{k}\right\|_{\infty}=M$. Since

$$
M=\left\|\varphi_{k}\right\|_{\infty} \leqq \frac{1}{m J_{k}}\left\|\varphi_{k}\right\|_{1}+V(\varphi)
$$

it follows that

$$
\frac{99}{100} M \leqq \frac{1}{m J_{k}}\left\|\varphi_{k}\right\|_{1}
$$

and

$$
\left\|\mathscr{P}^{N} \varphi\right\|_{\infty} \leqq\left(\frac{100}{99} e^{2 N_{0}} D^{N}\right)\|\varphi\|_{1} .
$$

Proof of Lemma 5.1. We fix $\varepsilon$ acceptable with regard to 5.1c and take $R=R_{1}+R_{2}+1$. (Note that if we had defined $\|\cdot\|=V(\cdot)+\|\cdot\|_{1}$, then $P$ could be unbounded. Take $\left.\varphi^{(k)}=\frac{1}{m J_{k}} 1_{J_{k}}.\right)$ 
Proof of Lemma 5.2. Let $N \in \mathbb{Z}^{+}$be as in Lemma 5.1, and let $E=E_{\beta^{N}}$ be the conditional expectation with respect to the $\sigma$-algebra generated by $\beta^{N}$ on $J$. Define

$$
\widehat{\mathscr{Q}}(\varphi)=\mathscr{P}^{N}(E \varphi)
$$

and

$$
\mathscr{Q}_{j}(\varphi)=\mathscr{P}^{N}\left(E\left(\varphi \cdot 1_{k \leqq j} J_{k}\right)\right)
$$

We claim that for sufficiently large $j, \mathscr{Q}=\mathscr{Q}_{j}$ has the desired property.

Sublemma 5.2a. $\left\|\mathscr{P}^{N}-\hat{\mathscr{Q}}\right\| \leqq \frac{1}{10}$.

Proof. Let $\theta=\varphi-E \varphi$, so that $\left(\mathscr{P}^{N}-\widehat{\mathscr{Q}}\right) \varphi=\mathscr{P}^{N} \theta$.

(i) $V\left(\mathscr{P}^{N} \theta\right) \leqq\left\|g_{N}\right\|_{\infty} \sum_{B \in \beta^{N}} V_{B}(\theta)+\max _{B \in \beta^{N}} V_{B}\left(g_{N}\right) \cdot \sum_{B}\left\|\theta \cdot 1_{B}\right\|_{\infty}$.

Since $V_{B}(\theta)=V_{B}(\varphi)$ and $\left\|\theta 1_{B}\right\|_{\infty} \leqq V_{B}(\theta)$, we conclude that

$$
V\left(\mathscr{P}^{N} \theta\right) \leqq \frac{5}{100} V(\varphi) \text {. }
$$

(ii) $\left\|\mathscr{P}^{N} \theta\right\|_{1} \leqq \sum_{B \in \beta^{N}} m B \cdot V_{B}(\theta) \leqq \frac{1}{100} V(\varphi)$.

(iii) The same argument as in 5.1c (Case 3 cannot occur) gives

$$
\varepsilon\left\|\mathscr{P}^{N} \theta\right\|_{\infty} \leqq \max \left\{\frac{1}{10} \varepsilon\|\theta\|_{\infty}, \frac{1}{100} V(\varphi)\right\} .
$$

To complete the proof of Lemma 5.2, consider an arbitrary $\varphi \in X$. Write

$$
\left\|\mathscr{P}^{N} \varphi-\mathscr{Q}_{j} \varphi\right\| \leqq\left\|\left(\mathscr{P}^{N}-\widehat{\mathscr{Q}}\right)\left(\varphi 1 \bigcup_{k \leqq j} J_{k}\right)\right\|+\left\|\mathscr{P}^{N}\left(\varphi 1 \bigcup_{k>j} J_{k}\right)\right\|
$$

The first term is $\leqq \frac{1}{10}\|\varphi\|$ by $5.2 \mathrm{a}$, and the second term is

$$
\leqq \frac{1}{5}\|\varphi\|+R\left\|\varphi 1 \bigcup_{k>j} J_{k}\right\|_{1}
$$

by Lemma 5.1. Since

$$
\left\|\varphi 1_{k>j} J_{k}\right\|_{1} \leqq\left(\sum_{k>j} m J_{k}\right) \cdot\|\varphi\|_{\infty} \leqq \frac{1}{\varepsilon}\left(\sum_{k>j} m J_{k}\right) \cdot\|\varphi\|,
$$

we have $\left\|\mathscr{P}^{N} \varphi-\mathscr{Q}_{j} \varphi\right\| \leqq \frac{1}{2}\|\varphi\|$ for sufficiently large $j$.

Note added after completion of manuscript. The author has heard that G. Keller and T. Nowicki recently obtained some related results.

\section{References}

[BC1] Benedicks, M., Carleson, L.: On iterations of $1-a x^{2}$ on $(-1,1)$. Ann. Math. 122, 1-25 (1985)

[BC2] Benedicks, M., Carleson, L.: The dynamics of the Hénon map. Ann. Math. 133, 73-169 (1991)

[BY] Benedicks, M., Young, L.-S.: Absolutely continuous invariant measures and random perturbations of certain one-dimensional maps to appear in Ergod. Theoret. Dynam. Sys. 
[BS] Bunimovich, L.A., Sinai, Ya.G.: Statistical properties of Lorentz gas with periodic configuration of scatterers. Commun. Math. Phys. 78, 479-497 (1981)

[C] Collet, P.: Ergodic properties some unimodal mappings of the interval, 1984 preprint

[CE] Collet, P., Eckmann, J.-P.: On the abundance of aperiodic behavior for maps on the interval. Commun. Math. Phys. 73, 115-160 (1980)

[DS] Dunford, Schwartz: Linear operators, Part I: General Theory 1957

[HK] Hofbauer, F., Keller, G.: Ergodic properties of invariant measures for piecewise monotonic transformations. Math Z. 119-140 (1982)

[J] Jakobson, M.: Absolutely continuous invariant measures for one-parameter families of one-dimensional maps. Commun. Math. Phys. 81, 39-88 (1981)

[K] Keller, G.: Un théoreme de la limite centrale pour une classe de transformations monotones par morceaux. C. R. Acad. Sci. Paris, t. 291, Série A, 155-158 (1980)

[LY] Lasota, A., Yorke, J.: On the existence of invariant measures for piecewise monotonic transformations. Trans. AMS 186, 481-488 (1973)

[L] Ledrappier, F.: Some properties of absolutely continuous invariant measures on an interval. Ergod. Th. Dynam. Sys. 1, 77-93 (1981)

[M] Misiurewicz, M.: Absolutely continuous measures for certain maps of an interval. Publ. Math. IHES 53, 17-51 (1981)

[N] Nowicki, T.: Symmetric S-unimodal mappings and positive Lyapunov exponents. Ergod. Th. Dynam. Sys. 5, 611-616 (1985)

[Ro] Rohlin, V.A.: Lectures on the theory of entropy of transformations with invariant measures. Russ. Math. Surv. 22(5), 1-54 (1967)

[Ru1] Ruelle, D.: Thermodynamic formalism. Reading, MA: Addison-Wesley 1978

[Ru2] Ruelle, D.: The thermodynamic formalism for expanding maps. Commun. Math. Phys. 125, 239-262 (1989)

[Ry1] Rychlik, M.: Bounded variation and invariant measures. Studia Math. LXXVI, 69-80 (1983)

[Ry2] Rychlik, M.: Another proof of Jacobson's theorem and related results. Ergod. Th Dynam. Sys. 9, 93-109 (1988)

[TTY] Thieullen, P., Tresser, C., Young, L.-S.: Positive exponent for generic 1-parameter families of 1-d maps, 1991 preprint

[Z] Ziemian, K.: On Perron-Frobenius operator and limit theorems for some maps of an interval. Ergod. Th. Rel. Topics II, Proceedings, Teubner Texte zür Math. 94, 206-211 (1987)

Communicated by T. Spencer 